\title{
Jean Barbin / François Bon : l'écriture monstrueuse, YouTube, l'édition du minuit de soi
}

\author{
Milène Tournier \\ Paris 3 Sorbonne Nouvelle
}

Sur la chaîne YouTube de l'auteur François Bon, surgit, le 8 Mars 2017, un invité troublant, nommé Jean Barbin (Bon, François, «La Chronique de Jean Barbin », Web, 09/08/2017.), aux traits étranges et déformés, et dont les premiers mots sont « Le monde est mal fait./ Comment peut-on s'accommoder du monde sans le casser./ Nous, les cassés d'avance./ Le monde est mal fait. Mais pas nous » (Bon, François, «Le monde est mal fait», Web, 09/08/2017) François Bon, alias Jean Barbin, s'adresse à nous, son visage fait irruption du centre de l'écran, cortex béant, présence cernée d'atrophie, visage sans corps et qui semble sortir d'un trou, d'un puits, arriver de loin, du noir, de la nuit, ramper jusqu'à la lumière. Jusqu'au 6 juillet 2017, chaque semaine, Jean Barbin sort de sa cave et nous parle, nous interroge. Monstre hebdomadaire qui, pour quelques minutes, soulève la trappe sous laquelle il est terré, passant de l'invisible au visible, du muet au proféré. La série, disponible sur YouTube et Vimeo, s'intitule «Logiques et autres liquides ». La première parole du monstre l'affirme monstre -il sera un "cassé d'avance »- en même temps qu'elle déporte et rejette la monstruosité sur « le monde ». Ce serait le monde qui serait " mal fait», défectueux, et non les monstres, les « mal faits », mal vus, mal regardés.

\section{La naissance du monstre}

«Un personnage, un clown, un double, une fiction, le monstre qu'on porte en soi» (Bon, «Jean Barbin tout nu.», Web, 09/08/2017): voilà comment François Bon décrit Jean Barbin, youtubeur noctambule et troublant. Jean Barbin, nous dit l'auteur, " est né à Montpellier, dans un appartement vide, un soir où ça n'allait pas très bien». Personnage issu de l'obscurité —François Bon dit ne s'éclairer qu'avec «la torche de l'Iphone»- et d'un " appartement vide », c'est un monstre qui sort, ou qu'on sort, la nuit, sorte de loup-garou 2.0. Il est à noter que la véritable apparition de Jean Barbin survient à l'occasion d'une intervention technique, d'une première métamorphose : «puis j'avais rajouté ces effets, sans aucune préméditation, Jean Barbin était né ». C'est sans doute ainsi que naissent les monstres, par « effet», un effet d'optique qui nous fera dévisager/ré envisager l'image, ce qu'on voyait. Incarnation déchirée, et déchirante, Jean Barbin voit le jour, « un soir où ça n'allait pas très bien » et prend forme, dans le creuset du noir et l'échine de la nuit, dans le secret d'un appartement vide. Cette première naissance est importante en cela qu'elle informe sans doute la manière dont nous devons recevoir ce monstre qui parle dans la nuit, depuis la nuit : peut-être est-il aussi à écouter dans la nuit, de nuit à nuit, d'un «ça ne va pas très bien » à « moi non plus ». A la déformation physique fait écho la distorsion sonore : le filtre audio du logiciel de montage vidéo Final Cut utilisé par l'auteur s'appelle d'ailleurs « monstre ».

Jean Barbin s'auto-décrit comme un monstre de la non-présence : 
Finalement j'ai préféré une absence à moi-même./ Il y a des choses qui ne vous plaisent pas./ Des situations dont vous ne savez comment vous en sortir./ Alors j'avais choisi l'absence à moi-même./ Je dormais. Je dormais beaucoup, longtemps./ Puis ça se calmait./ Le temps étale les choses./ Je reprenais. C'est étrange comme les autres remarquent peu votre absence à vous-même, lorsque vous reprenez. Ne posent pas de question./ Alors, émotion, peine, choses manquées, trucs ratés, absence à moi-même./ J'ai lu, beaucoup lu, sur ces animaux qui font le mort, la libellule, des oiseaux, le hérisson même, quand ils se sentent menacés./ J'étais ces insectes tombés morts, provisoirement morts./ Puis je reprenais./ Mais le problème, c'est qu'on s'y fait, on s'y fait très bien, à l'absence à soi-même./ Donc je faisais durer. Revenir: mais revenir pourquoi, je me disais à moi-même./ Et c'est alors que la transformation a commencé. (Bon, « Jean Barbin, absence », Web, 09/08/2017)

Pourtant, et alors même que ce monstre semble assommé d'immobilité, il n'a de cesse de commencer, comme autant de débuts avortés de récits, un phœnix narratif, une fiction reptilienne dont la queue repousserait toujours. Son absence à lui-même devient son mode de visibilité, d'apparition, et d'être.

\section{Le monstre et nous}

Le monstre, comme figure, pourrait se caractériser par l'intrusion, l'effraction, qu'il commet. Déflagrations qui nous somment de les regarder, ces figures-là, monstrueuses, feignent de toujours surgir, hors-sol, hors-temps, semblent arriver d'un ailleurs. À ce jeu du trop-montrer, les monstres nous aveuglent, masquant le support auquel ils s'arrachent. Jean Barbin, sorte de clown blanc un rien dégénéré, qui aurait étalé sa pâleur candide (au sens étymologique), laiteuse à même les rougeurs et tuméfactions de ses joues, philosophe faussement naïf, concasse les mots jusqu'à l'absurde, jusqu'à l'envers. Poète de l'existentiel, Jean Barbin s'inscrit pourtant dans une certaine continuité avec les figures dont François Bon a peuplé ses romans : marginaux ou précarisés, ceux qui ont peu la parole, ceux qui « ne comptent pas », ceux des bords des villes, des banlieues de la société, il en serait l'émanation même, une émanation sans contour, où ne subsisterait plus que, irrésistible, cette impression vague de désarroi, d'être à côté du monde, ou que le monde se passe à côté de soi.

Car la créature de François Bon a ceci de singulier dans la galerie des monstres qu'il est « gentil », profondément " gentil». La tête d'un monstre, quand les monstres sont doux, et même leur voix. Sorte d'être hydrocéphale, d'ombilic des limbes, pour reprendre un titre de Antonin Artaud, qui a lui aussi enfanté d'autres monstres singuliers, Jean Barbin ne soliloque pas, il sollicite notre écoute et va même jusqu'à nous interroger, voire nous conseiller. Ainsi, dès la deuxième vidéo :

«Je rachète les peurs », Jean Barbin se fait dealer de nuit, petit trafiquant, vendeur à la sauvette, pour nous sauver, se sauver lui-même, rabatteur de l'ombre: «Le problème du monde c'est la peur./ Le problème de chacun c'est la peur./ Je propose un remède contre la peur./ Je vous rachète votre peur./ Quand j'ai toute la peur, je l'annihile./ Alors le monde vivra plus libre./ Chacun vivra plus libre./ Je vivrai plus libre./ 
Vendez-moi votre peur./ Je rachète toutes les peurs. (Bon, « Jean Barbin , Je rachète », Web, 09/08/2017)

Dans la cinquième vidéo de la série, « Sortir son monstre », Jean Barbin se fait même coach de vie, ou de survie : « Il faut convoquer le monstre de soi./ Le monstre de soi n'est pas visible à soi./... Il faut entrer en soi, chercher le monstre de soi, et faire sortir le monstre de soi./ Ici parle le monstre de moi./ .... Ouvrez, ouvrez le dehors au monstre de soi, si soi c'est vous » (Bon, «Jean Barbin, Le monstre », Web, 09/08/2017).

Etre aquatique, baignant dans son jus de noir, être amphibien d'avant les bras et les jambes, dans son aquarium de noir, Jean Barbin s'extirpe de la solitude, pour créer un lien, un lien ombilical, un lien qui tiendrait presque à des paramètres techniques : les réglages de la voix, entre effets, résonances, échos, jeu de canon, répétition, déformation, ralentissement, réverbération, sont tels qu'ils induisent en retour une écoute du dedans. C'est la voix utérine qui courbe et plie l'oreille dans l'inclinaison nécessaire pour la recueillir, on l'entendra comme du dedans, voix stéthoscope à cogner aux parois. Des paroles à peutêtre écouter avec un casque, entendre depuis l'intérieur de la tête, qui ne viennent pas du dehors, qui montent dedans. La parole provoque déjà l'écoute qu'elle espère, et vidéo et oreille feront ensemble un coquillage. Jean Barbin se présente alors comme un " appel au secours » : "Je suis un appel au secours vivant./ Je suis un appel au secours perdu./ Je suis un appel au secours inaudible. / Je suis un appel au secours désespéré./ Je suis un appel au secours collectif./ Je suis un appel au secours impossible./ Je suis l'appel au secours de tous les appels au secours./ Au secours!» (Bon, «Jean Barbin, Je suis », Web, 09/08/2017). Jean Barbin se présente comme une toute-sollicitation, comme un appel, la voix de quand on appelle, dans le noir. Comme cela arrive à certains d'entre nous d'appeler le Samu la nuit, en pleine nuit, et de dire son nom, dire son adresse, et cela dans le noir, et qu'il y ait un téléphone ne rend pas la chose plus réelle. Pourtant la voix attestera qu'il y a un monde hors de la pièce. Jean Barbin peut sembler cet appel nocturne, de qui est seul face au mur d'angoisses, que le flanc de la nuit épaissit. Comme les monstres tapis sous le lit le jour attendent l'obscurité pour sortir.

Visage sans âge, visage de tout temps, entre petite enfance, avorton, et grande vieillesse :

On gagne à visiter le sans âge de soi-même./ Si tôt existe en soi le sans âge de soi-même./ On enlève ses peaux, ses yeux, on se soulève le crâne, on s'écarte le dos, on pousse la cage des os./ Il est là le sans âge de soimême./ ... Cherchez-le dans les vivants, dans les passants, le sans âge d'eux-mêmes./ Et puis regardez-le en face, le sans âge de vous-même./ Laissez-le parlez pour vous, le sans âge de vous-même./ Une fois j'ai décidé cela: ne plus être que le sans âge de moi-même./ Entrer en lui, et n'en plus revenir./ Y disparaître comme lui d'avance avait disparu en nous: le sans âge de soi-même. (Bon, « Jean Barbin, Le sans âge ", Web, 09/08/2017)

L'écriture, comme tentative de dépasser l'âge, et le sens que prend la vie parfois, le pli qu'elle attrape, le virage que le dehors lui a imprimé. Choisir l'envers pour résister au pli. Car c'est bien là le grand problème du monstre, qu'on le réduise à ce qu'on en voit, ou croit en voir : 
Il y en a, quand ils me voient, ils rient./ Moi je ne ris pas de moi-même./ C'est l'âge qui vous use le dehors./ Dedans ça ne change pas./ Dedans on a les yeux clairs./ Mais ce qui vous est pris dehors ça marque aussi le dedans./ C'est là qu'on a peur : dehors ne compte pas mais quoi, si ça vous mange aussi le dedans ?/ Alors moi je montre : je montre ce que prend l'âge au-dehors./ Et je dis les mots du dedans, les mots qui ignorent le dehors./ Mais qui savent comment le dehors mange le dedans. (Bon, « Jean Barbin, Je n'aime pas », 09/08/2017)

Le trop-visible du monstre le dessert, parce que le trop-visible est toujours plus laid que ce que nous sommes, le plus laid de nous dehors, qui prend le vent. Dire alors la monstruosité et, simultanément, la banalité de vieillir. La monstruosité et la banalité-mourir, devoir mourir, un jour se taire, un jour aucun mot dedans-dehors.

\section{Le monstre du monstre}

Sur la page de présentation des chroniques de Jean Barbin, François Bon confie : «Il est probable que l'accompagnement actuel de ma vieille maman dans les ténèbres de l'Alzheimer ait contribué à cette recherche ». Derrière ce monstre-ci, visible, un autre monstre, invisible, la figure maternelle, étrangère à elle-même, égarée dans d'autres ténèbres. Ce serait par la nuit du sens, qu'on pourrait rejoindre une mère malade. Un autre "accompagnement», plus lointain, et qu'on fait seul, sans dire, comme on prie parfois pour quelqu'un et sans l'en avertir. Un monstre qui console, ou du moins soigne un autre monstre. Qui accompagne en tous cas, qui calque son pas sur celui de l'autre, son rythme, sa cadence. Entrer, pénétrer dans ce rythme abîmé (au sens premiers des abîmes) implique alors de s'arracher à celui des villes, des semaines, des heures. Comme le temps passe étrangement, dans les maisons de retraite, on en sort, la ville foudroie : qu'elle existe encore !, qu'elle existe encore. Et le temps qu'il faut, pour se ré-accomoder, pour revenir à. Parce que parfois c'est difficile de rester, de demeurer auprès, et aussi longtemps qu'on se l'était promis. Jean Barbin semble alors faire le même trajet, peut-être, que celui auquel la personne atteinte d'Alzheimer est astreinte : un enfouissement dans le noir, s'enfoncer et s'absenter à. Les capsules vidéos ressemblent alors à des crustacés, recroquevillées sur le noir et autour du silence. Des vidéos vertiges, des vidéostunnels, pour atteindre un hors-ville, hors-sens, un état d'incertitude, comme dirait Claude Régy. Quand c'est le même qui est destitué, la certitude du même, et la reconnaissance. Qu'une chose soit, et qu'elle soit la même chose demain, qu'on pourra la reconnaître. Les mots, défaits de la gangue du sens, redeviennent apparitions exorbitées, fulgurances, et c'est par le son qu'on s'en souviendra, par le plaisir de l'éboulis de la gorge au dehors.

Quand la pathologie est aussi une maladie du lien, qui affecte deux au lieu de n'affecter qu'un, qui brouille le temps, et comment se retrouver soi, avoir foi dans l'assurance d'un soi, d'une bio-graphie, quand l'autre se perd, qui plus est quand l'autre est une mère ? Ce que ce serait, d'écrire sous Alzheimer comme on dirait sous drogue? Et pointant vers l'absence, le visage qu'auraient nos textes alors ? Ces vidéos en flottaison semblent alors ce moyen trouvé, pour écrire depuis cette nuit du temps, d'écrire en reculant. 
Cette solitude, des mots retournés dans la nuit, est aussi une liberté. Écrire sans plus de regard, écrire sans épaule et personne pour regarder pardessus. La vidéo, dans sa forme même, est fidèle aux conditions de sa fabrication, le fond noir singeant la nuit. L'alter-ego monstrueux émerge de ces nuits d'écriture. Le média de l'écriture, dans la claustration qu'il applique à la figure, ne s'accommode du média de la vidéo (et, même, le réclame) qu'à la condition que celle-ci offre la même liberté que l'écrit. "Les vidéos ont été réalisées avec un Canon 80D sur trépied, objectif Sigma Art 18-35, micro large membrane Røde NT1-A sur Zoom H4N ou Røde VideoMic sur caméra, plugin 360FX sur FinalCut pour l'effet sphérique. La plupart sont écrites, filmées et montées en 30 ', le soir vers $23 \mathrm{~h}$, comme principe d'action de cette série. » Ici, filmer, se filmer, s'auto-filmer a une vocation double, et contradictoire : extraire le corps de la solitude de l'écriture, mais aussi reproduire les conditions de celleci. Ce n'est pas la solitude qu'il faut exhiber, c'est la solitude de l'écriture, avec l'écriture, par l'écriture, la solitude qu'on choisit parce qu'elle a fait écrire, préserver dans la vidéo le hors-de-toute-honte de ces heures où l'écran de l'ordinateur et un quart de lune se disputent le noir. Les mains sur le clavier, à ce moment-là, on est seul à vivre, et ce n'est plus nous qui sommes invisibles, mais le reste, la ville, les gens, les autres. Il s'agirait de passer de filmer la nuit à filmer de nuit. Filmer de nuit c'est filmer du silence, les autres dormant.

\section{La genèse du monstre}

Les vidéos de cet auteur, observateur patient et passionné des villes, comment elles bougent, comment elles vivent, "se font et se défont", révoquent ici pourtant tout référentiel, se nicher ou se cloîtrer dans un espace abstrait, à la lisière des villes, dans les entrailles des villes, quand plus de ville du tout. Cellules monacales où s'en-gironner soi-même. Une solitude totale, puisque hors monde hors temps, hors espace, hors les choses qui font qu'on est au monde, qu'on peut se situer dans le temps et l'espace à la vue de tous Jean Barbin n'a de cesse, dans ces vidéos, de raconter et broder son histoire, sa genèse, de quel noir il provient, de quoi il nous prévient. Les titres des vidéos sont largement auto-référentiels ou monstruo-référentiels : « La voix enfant», " Malheureux parmi les hommes », « Je fus trou », « Rétrospectivement », « Où je suis ? », « Quand ils m'ont découvert »...

François Bon semble alors démontrer le mystère de la formation, ou floraison, du personnage, l'avènement du monstre. Il est d'ailleurs à noter que, sur la page de son site dédié à un atelier d'écriture qui propose d'explorer la notion de personnage, l'auteur «met cet atelier sous le signe d'Alfred Kubin» (Bon, François, « atelier été 2017 | et si je vous dis « personnages » ?», Web, 09/08/2017), et place en exergue une gravure de l'artiste qui dépeint une figure larvaire, au ventre protubérant et aux membres squelettiques, sortie de son trou, recluse et presque fluorescente à la manière des vers luisants. Comme si tout personnage recelait son monstre. L'auteur explique d'ailleurs : «Jean Barbin s'est peu à peu révélé à moi-même, comment et où il vit, comment il parle» (Bon, François, "Jean Barbin tout nu »). Ce terme de révélé est important, qui associe d'emblée la figure monstrueuse à la lumière, ce qui se découvre, ce qui se cache ou se montre. 
Jean Barbin est éminemment lié à l'invisible d'où il s'extirpe. Sorte de Bob de la série Lynchéenne Twin Peaks, issu de sa « Loge noire », personnage construit comme une vision, presque déjà une hallucination. Jean Barbin, à son tour, semble un personnage cousu ou retourné sur son envers, qui parle son enfance. Ainsi dans la vidéo « Le placard de soi-disant », Jean Barbin se figure en orphelin remisé au placard :

Je fus mis dans un placard à l'âge de trois ans./ J'y fus mis./ Je ne sais pas pourquoi./ Je ne sais pas qui./ J'ai tout appris moi-même, du dedans du placard./ Je n'ai jamais rien vu du dehors, longtemps (on m'a dit qu'il y a un dehors)./ Qu'est-ce que j'ai manqué ?/ Cela m'a protégé./ Mon vide dedans est plus grand./ On s'encombre moins du monde. De dehors. / On finit par l'aimer, son placard. On l'aime, son placard./ Parfois, on me sort de mon placard. On me fait aller dans des endroits, on me met dans des lumières./ On me dit de parler. Des fois./ Je n'aime plus tellement sortir de mon placard./ Pourquoi ils m'ont mis dans ce placard ?/ Qui m'a mis dans ce placard ?/ Et pour les autres, c'est pareil - c'est quoi, le placard de vous-même? (Bon, « Jean Barbin, sortir son monstre», 09/08/2017)

Enfant du placard, enfant du noir, qui parle depuis le placard, comme ces enfants albinos qu'on retire de la lumière, qu'on cache du jour, à la manière de certains personnages de marginaux, dans les pièces de Koltès, qui ont « leur peau retournée sur l'envers » (Koltés, Quai Ouest). Comme le personnage d'Abad dans Quai Ouest, monstre muet, monstre dans sa graphie même, qui ne s'exprime qu'entre parenthèses, marginalisé depuis l'espace de la feuille, personnage noir, dont Koltès dira qu'il n'est pas « un personnage en négatif au milieu de la pièce, mais c'est la pièce qui est le négatif du Noir », inversant et renversant alors définitivement l'alternance jour/nuit, centre/marge, monstre/norme. Sorte d'enfant caché, poussé, grandi sous les escaliers, Jean Barbin devient alors la figure même de l'enfant sauvage, celui qui a appris à parler tout seul, entre ses doigts, contre la paume de sa main, en mettant ses mains en cornet devant sa bouche, pour sentir sa propre buée et jusqu'à ce que ça fasse un son. François Bon semble alors nous faire accéder à la poésie de celui qui a appris à parler dans le noir, et entre les murs, sans recours à l'imitation, et demeuré dans une forme de stade anténatal du langage. C'est comment, parler, quand on n'a pas appris à ? «Et pour les autres c'est pareil, c'est quoi ? », nous interroge le monstre, et c'est peut-être ici la question existentielle de l'existence de Jean Barbin, le monstrueusement pareil de chacun.

Observant le jasi des enfants, et discernant le proto-langage qui affleure dans ces soliloques-babils, Aliyah Morgenstern analyse de quelle manière une énonciation du « je » propre à l'enfant advient dans le langage (Morgenstern). Elle observe la façon dont s'installe « le processus de mise en place du marqueur de première personne je». Loin d'être linéaire, cette trajectoire vers le «je » passe en fait par des formes multiples d'auto-désignation, et un recours à d'autres pronoms personnels. Se désignant dans un « tu » réprobateur, un « il » coupable, un « vous » d'auto-félicitation, l'enfant, dans le saisissement qu'il fait de sa présence par les pronoms personnels de l'Autre, copie et s'approprie des situations qu'il cherche à rejouer : être grondé, puni, admiré... Jean Barbin semble, lui, baigner dans une eau de langage, selon le titre de la série « logiques et autres liquides », un état placentaire du langage, qui n'exclut pas le silence, 
et qui ne s'en écarte pas, qui sans cesse y retourne. Une genèse qui ressasse sa genèse, un monstre prématuré dont on trame a posteriori la naissance, qu'on recuit, qu'on achève, et la vidéo comme une couveuse. Il n'a de cesse, pourtant, à son tour, de jouer avec les pronoms, s'amusant avec sa voix à révoquer, invoquer ou convoquer, comme on joue à parler fort ou parler bas, dans le noir, pour voir jusqu'où va sa propre voix :

Parfois la nuit, avant le rêve, je marche longtemps dans la nuit de moimême./ La nuit de moi-même est un ciel, mon ciel, une sorte de ciel./ Dans cette nuit sous le ciel, marchant dans moi-même, alors j'entends une autre musique./ Ce n'est pas une musique pour instrument de musique. C'est une musique musique./ Une musique du dedans de vous-même sous le ciel de la nuit, votre nuit, la nuit quand vous marchez dans le dedans de vous-même./ Écoutez. C'est ma musique. Je la donne./ Elle n'est pas si facile à entendre./ Ceux qui savent marcher dans le dedans de soi-même, le dedans d'avant le rêve, ceux-là et celles-là ils et elles l'entendront./ Moi je suis sûr que vous savez l'entendre./ Et j'entendrai la vôtre, aussi. (Bon, « Jean Barbin, Musique », Web, 09/08/2017)

Le mot semble, pour Jean Barbin, compter autant, voire plus, que le référent qu'il désigne. Plus : le proférer annule le voir. Le langage perd sa dimension mimétique et semble substituer au regard sur l'objet le scintillement médusant du logogramme du signe. Se faisant tout-sensuel, le langage de Jean Barbin ne s'interpose plus entre le regard et le dehors, il prend presque la place du monde, par l'excitation du mot. On n'est pas pourtant dans la " glossolalie ", ou en tous cas pas dans la glossolalie folklorique. Il s'agit tout de même de faire grincer la voix des os. De passer de la voix qui convoque à celle qui invoque, qui révoque, qui dévoque presque, qui déparle. Parler dans le noir, proférer dans la nuit. Les conditions mêmes sont importantes, qui font en même temps qu'elles sont. Quand la ville éteinte, la ville close, on peut être au langage comme en forêt, sans plus de rappel à la civilisation et au calendrier.

Dans son article Autoportraits et glossolalies : la douloureuse musique d'Antonin Artaud, Camille Murando établit un parallèle entre les autoportraits dessinés par l'auteur et son usage de la glossolalie, et repère que les dessins de Artaud procèdent d'une même technique de déformation du tracé que la glossolalie applique au langage, le trait se rapprochant du craché-vociféré, expulsé. Elle cite Antonin Artaud : « Je me suis désespéré du pur dessin. Je veux dire qu'il y a dans mes dessins une espèce de morale musique que $\mathrm{j}$ 'ai faite en vivant mes traits non avec la main seulement, mais avec le raclement du souffle de ma trachée-artère, et des dents de ma mastication » (Artaud 266.) Mais précisément, si l'autoportrait dessiné tient de la glossolalie, c'est peut-être parce que la glossolalie est elle-même un autoportrait, celui du monstre de soi. Loin d'être alors un langage-cri qui se projette, la glossolalie s'arrache à l'intime, dans cette « invention » de soi qui est peut-être le portrait de soi. La langue de Jean Barbin, comme un symptôme béant, à ciel et cœur ouverts, attendrit, comme on le dit à propos d'une viande, en cela qu'en même temps qu'elle pose une question, elle paraît aussi poser une musique.

Jean Barbin est bien un monstre issu de l'invisible, qu'on découvre. Ainsi dans la capsule intitulée « Quand ils m'ont découvert», il se figure en rat de laboratoire, objet d'expériences médicales sordides, en papillon écarquillé, 
punaisé à l'image. Fossilisé, pétrifié, le visage entier comme un hérisson tétanisé :

Quand ils m'ont découvert, il y eut de nombreux articles de journaux. Pensez, un homme fossile. Découverte d'un homme fossile. Ça changeait parait-il notre connaissance de l'homme et de son histoire : un fossile de l'homme des temps modernes. Un fossile qui nous parvenait directement de maintenant à maintenant. Alors ils m'ont mis dans un laboratoire. Ils m'ont fait des IRM, des rayons. Des prélèvements aussi, et sans me demander mon avis : un bout de ci, un bout de ça. Vas-y que je te prends un bout de cervelle, vas-y que je te gratte la moëlle des os, vas-y que je te regarde dans les oreilles. Ils m'ont fait des datations, et datatations, tout ce qu'ils savaient : carbone 14, 17, 24, 35, 114, rayonnement gamma, diffraction des ultra $X$, érosion électro-chimique. Oui, j'étais bien un homme de maintenant. Oui, un fossile d'homme de maintenant pouvait se révéler effrayant sur la condition des hommes de maintenant et notre régression depuis l'homme du passé. On m'a testé la mémoire : je me souvenais. On m'a testé le raisonnement : je raisonnais. Puis lentement ils ont injecté leurs produits. Là j'ai dit non. Faites-moi ce que vous voulez, faites-le sur vous-même en même temps. Ils m'ont dit : - Ah, tête de cochon, caractériel, et ils ont débranché leurs appareils, arrêté les transferts, m'ont dit d'attendre. D'attendre là dans le noir. C'est depuis, là, que j'attends. Fossile de maintenant, et plus personne qui ne veut le voir, ni savoir. (Bon, «Jean Barbin, Quand ils m'ont découvert», Web, 09/08/2017.)

Le monstre passe de l'invisibilité à celui qu'on examine, qu'on scrute, jusqu'au dedans, celui auquel on refuse toute possibilité de mystère, de réserve, dont on étale l'anatomie à même une planche, qu'on autopsie vivant, qu'on fait passer du noir à la lumière crue, sur lequel on braque une torche, qu'on le dépouille jusqu'à le rendre transparent.

\section{Monstrueux support}

Dans la vidéo « Je fus trou », Jean Barbin se (dé)figure en trou, béance vivante, excavation incarnée :

Je fus trou longtemps. Les paroles tombaient au fond et ne ressortaient pas. Donc on ne m'entendit pas, jamais. Cela dura longtemps. Puis finalement le trou de paroles se combla. En envoie-t-on, des paroles que personne n'écoute, qu'elles retombent et que ça comble aussi le puits. Quiconque avance avec les yeux ouverts sur la nuit du monde est un trou de paroles mortes. Puis un jour ça déborde. Alors voilà, j'ai ouvert ma bouche sur la nuit du monde. J'ai ouvert mes yeux sur la nuit du monde. Puis j'ai renvoyé tout cela qui attendait dans le puits, qui a mûri dans le puits. Je fus trou, mais maintenant mon trou parle. Et le monde peut bien tourner le dos, s'il n'est qu'un trou, lui aussi, la somme de tous les trous des mots. Les yeux se détournent, quand tu parles, ignore les yeux. Les corps fuient dans la ville, quand tu parles, ignore qui te fuit. En toi le besoin de voir, en toi de disposer de tout ton noir, de vider ton noir. Etre 
trou, pour mieux s'entendre. (Bon, «Jean Barbin, Je fus trou, Web, 09/08/2017)

Jean Barbin semble alors s'adresser à nous depuis les caves de YouTube, les souterrains, un vrai darknet, les catacombes, l'outre-tombe du web. L'arrière-plan noir semble signer un éternel trou, sans fond, sans fin, une grottegorge d'où ne pourra s'extraire que la tête, le corps demeurant, lui, aspiré dans les abysses.

François Bon dit que « les réglages de sa voix sont désormais toujours les mêmes, mais les distorsions du visage ; chaque fois une violence de hasard » (Bon, «Jean Barbin tout nu »). Ces altérations imprimées au visage, à l'image du visage, qui font du visage une toute-grimace, ne sont pas sans rappeler les peintures de Francis Bacon. Une (con)torsion-image appliquée à la gueule entière. Dans Logique de la sensation, Gilles Deleuze dit à propos de Bacon qu'il « peint la viande sans les os », et c'est bien en effet cela que permet l'effet infligé à l'image, qui fait capituler l'os, abdiquer le crâne comme forme, qui rend mou ce qui était dur. Le visage redevient cette forme sans forme, labile, glissante. La lacération du visage devient alors celle de l'image même, cicatrice bouffie, comme autrefois les pellicules pouvaient se consumer dans le projecteur, et qui distille des lambeaux de disparition et de dégradation dans l'instant même de la pure présence. Une dégénérescence-image. Le visage, mondé, pelé, levé, devient la ligne d'écriture même. La malformation imposée à la figure fait entendre les mots différemment, leur donne, étrangement, plus de tendresse encore, on voudrait le câliner, ce Jean Barbin. Tous ces visages qu'on a, tous ces visages qu'on pourrait, une fois dissoute la ligne visage, la forme visage, qui le tenait rond, qui le tenait ensemble, qui le tenait un, le visage fondu, rendu à l'étalement, qui se dépose sur le noir comme la cire coule. A la manière des taches d'encre du test de Rorschach, qui vont chercher dans notre inconscient, là où l'esprit à son tour n'est plus que noir, tache d'encre coulée sans nous, coulée avant nous, coulée fotale, et qui nous demandent de chercher quand même la figure, de voir au noir, de voir dans le noir, de voir avec le noir, comme c'est finalement la lumière qu'on retient des peintures de Pierre Soulages, quand le noir n'est plus qu'un palimpseste de torches et de mains, des mains qui ne sont pas là pour cacher la lumière, mais pour la protéger plutôt, ne pas l'user.

Le visage-Barbin devient alors une carne qu'on n'aurait pas détourée, une simagrée viandeuse :

Je me mange moi-même./ C'est l'avantage de vivre dans une seule pièce et dans le noir./ On se mange soi-même, ça repousse./ On est autosuffisant./ C'est de la viande hors sol./ La viande qu'on est soimême./ Se nourrir de soi-même est une solution de survie possible pour l'humain./ Il faut juste une obscurité suffisante, et que chacun supporte l'obscurité de lui-même./ Il faut juste que chacun soit dans sa boîte appropriée, vive dans sa pièce noire, accepte de n'en pas sortir./ Si on sort, ou si trop de lumière, ça ne repousse pas./ ... Dans le noir et dans mes murs, je mange ma viande et tout va bien. (Bon, « Jean Barbin, Mange ta viande », Web 09/08/2017)

Loméchuse (pour reprendre un titre d'Emma Santos, autre auteure du monstre en soi, de soi) autophage et auto-régénérante, on pourrait vivre 
toujours, vivre indéfiniment, si hors du monde. Auto-régérenescence ou autoparasitisme, quand, se confiant au noir, confiant sa vie au noir, on se met aussi en position, finalement, d'en être exclu. Jusqu'au symphilisme, et, dans la dernière vidéo "La page morte que nous sommes ", jusqu'à manger sa peau, son cuir, ses propres pages (Jean Barbin dévore des pages à vue), pour, finalement, retourner au noir.

La camera oscura de la vidéo s'apparente alors à une immense idée noire :

J'ai des idées noires./ De mes idées noires je compose une boisson, noire./ C'est assez simple : prenez vos idées noires, sortez-les de vous-même, pressez-les dans un compotier (inox, si possible)./... Puis réchauffez lentement, à température de corps, en tenant le compotier contre votre ventre./ .../ Tournez, lentement, longtemps./ Puis mettez en bouteille - de toutes petites bouteilles suffisent, des flacons, des fioles, fiasques, burettes./ Quand vous viennent d'autres idées noires, celles qui font mal, celles de la douleur des autres, de la douleur du monde, du mal fait à la Terre, de l'injustice faite aux autres ou à vous-même, prenez le flacon, fiole, fiasque, bouteille et versez trois gouttes./ Les idées noires, longtemps réchauffées contre le ventre dans le compotier inox, dissolvent les mauvaises idées noires./ C'est tout./ ... / Luttez contre vos idées noires./ Luttez contre vos idées noires avec vos idées noires./ Reste le besoin d'un compotier inox. (Bon, « Jean Barbin, J'ai des idées », Web 09/08/2017)

Le noir qui séparait devient ce qui fait lien, qu'on pourra retrouver partout et toujours, il suffira d'éteindre, cette possibilité du noir, du faire-le-noir en soi et dehors de soi, qu'on porte avec nous, sur nous. La vidéo prend des allures d'échographie, où on viendrait scruter le bébé, vérifier si «tout est normal ». L'image-matière prend corps dans la matérialité du noir et des couleurs-visage. Images matières, comme on dirait des selles, et impossible matière première, quand c'est la mémoire même qui est touchée, endommagée.

C'est peut-être parce que le monstre, et malgré son étymologie, rechigne tant à la lumière, à se hisser hors de l'invisible, comme le cafard de Kafka se terre en chambre, qu'il faut « forcener le subjectile » ainsi que le disait Derrida à propos d'Artaud. Évacuer l'alentour, plonger dans l'abstraction, et agresser le visage-support, pour préserver peut-être " quelque chose noir», ainsi que pourrait l'écrire Jacques Roubaud, la voix-poésie, quand elle touche encore le silence.

En requérant le souvenir, l'idée d'Artaud, la figure d'Artaud - dont l'œuvre entière vise pourtant à anéantir la figure, à broyer la face de la figure, comme le rappelle Evelyne Grossman dans La Défiguration, Artaud, Beckett, Michaux, attribuant aux trois artistes le geste commun de sabrer la certitude de l'identité : «Sous ce mot de défiguration, on tentera de suivre le mouvement de déstabilisation qui affecte, dans les textes modernes, la figure : mise en question inlassable des formes de la vérité et du sens » (Grossman 2004)_, on en appelle en fait une pensée plus large jetée sur la vie et sur l'art, simultanément. «L'aliéné authentique» (Grossman 2003) a porté, endossé, incarné sa pensée. Au point qu'on peut, yeux fermés, arrimer sa propre nécessité à la sienne. S'il a invectivé le support, "forcené le subjectile » (Derrida, 1986,) le visage de sa pensée, lui, persiste. Comme un subjectile ultime, intouchable celui-là, sa 
pensée enfouie dans la pensée de l'autre. Jacques Derrida rappelle que le geste destructeur d'Artaud recèle une vitalité qui entend donner figure dans le temps même de la perforation, dans l'instant même de la brûlure : " Comme ce support est là pour recevoir passivement les formes et les figures, le subjectile devient une sorte de X anonyme contre lequel Artaud s'emporte. Tout se passe comme si Artaud voulait lui faire prendre figure » (Derrida "Artaud et ses doubles » n.p.).

Mais précisément, Jean Barbin réussit peut-être à faire oublier jusqu'à son visage. Et si l'on n'a pas rencontré François Bon « en vrai» (ce qui est mon cas), on se trouve, à la fin de la série des 37 vidéos, bien incapable de se le figurer, sinon les lunettes, les cheveux blancs. Comme le souvenir d'une chose qu'on aurait oubliée. La trace de juste une trace. «j'ai été construit selon un principe du rêve » nous dit Jean Barbin. François Bon, lui, écrit : «Et dans le rêve, on reconnaît souvent le personnage sans déchiffrer ses traits. C'est même bien mystérieux la façon dont on découvre son propre visage dans le rêve » (Bon, " NSup », Web, 09/08/2017). Il s'agirait alors de passer de l'écriture personnelle à l'écriture en personne et, finalement, à l'écriture du personne de soi, du personne en soi, du soi quand il n'y a plus rien autour, du soi quand c'est le rêve, qui entoure le visage. La vidéo entière semble alors un rêve, un rêve comme mis en bouteille, en flacon, et le visage à flotter dedans comme un insecte. Avoir l'onirisme pour bunker, une opaque casemate pour chambre à soi.

Jean Barbin semble en effet piégé dans sa propre apparition, coincé dans un tunnel sans queue ni tête :

Je vivais comme tout le monde. Je ressemblais à tout le monde. J'étais tout le monde./ Je pensais comme tout le monde, je faisais comme tout le monde./ Alors ils ont commencé à creuser ce tunnel./ Là, en pleine rue, face ma fenêtre./ [...] Un jour je leur ai demandé : « Un tunnel pour où, un tunnel qui va où. » Ils ne savaient pas, les gars./ On peut bien creuser un tunnel d'abord, et voir où il débouche ensuite./ Il y avait des palissades et des grillages. Ça s'enfonçait profond. Ils ont mis du carrelage, mis des éclairages, et retiré les grillages./ Ce jour-là ils m'ont dit : " C'est prêt ». [...] / Alors je suis entré dans le tunnel, j'ai compris qu'ils rebouchaient l'entrée./ Maintenant je suis là./ Depuis longtemps, je suis là./ Depuis le fond du tunnel, je vous parle, je vous dis ça./ Si c'est bien, si c'est mal, je ne sais pas. / Ça ne m'avait pas surpris tant que ça./ Il était pour moi, le tunnel, et je ne le savais pas. (Bon «Jean Barbin, Vint le tunnel», Web, 09/08/2017)

La vidéo, espace d'apparition, devient alors aussi espace de claustration, prison, et il n'y aura pas d'ailleurs, nulle ville à mettre en arrière-plan, nul fond autre que le noir où dessiner le visage. Comme si, entre le monde et le monstre, il en fallait au moins un qui soit invisible, qui disparaisse.

\section{L'envers du monstre}

Jean Barbin, dans sa poésie perplexe, nous renvoie les questions philosophiques des enfants, de la couleur qu'ont nos paupières, si roses ou si noires, et du bourdon dedans que j'entends si tu l'entends aussi ou si le bruit n'est qu'à moi, d'où commence et ou finit mon corps, d'à partir de quel moment, de quel endroit, mon corps devient le monde et inversement. Jean Barbin est 
invisible, ou bien il demande un regard autre, un " œil arrière ": "Certains disent le troisième œil, moi je l'appelle l'œil arrière - ce que voit l'œil depuis l'intérieur arrière de la paroi du crâne./ Savez-vous vous servir de l'œil intérieur? Ça s'apprend, figurez-vous./ Celui-là voit autrement. C'est celui-là qu'il ne faut pas aveugler./ Donc vivez d'un œil. Mais l'œil arrière » (Bon, «Jean Barbin, De l'oeil arrière ", Web, 09/08/2017). Comme on fait basculer une caméra du mode portrait au mode selfie, l'invaginant presque.

Jean Barbin réalise en fait le défi de filmer en arrière, de filmer en rétrogradant, et la caméra fait office alors de plan de régression :

Quelle pitié ce que je suis devenu./ Le mot rétrospectivement./ On ne constate que rétrospectivement ce qu'on est devenu./ Alors on s'effraie : tu es donc devenu ça ?/ D'autant que c'est progressif. Regardez vos ongles, ils poussent. Regardez vos cheveux, ils poussent. Mais on ne le voit pas. Et ça continue même quand vous êtes morts./ ... Marche arrière, marche arrière !/ Mais qui donc aurait le bouton pour la marche arrière ?/ Évitez, évitez de devenir. (Bon, «Jean Barbin, Rétrospectivement», Web, 09/08/2017)

Le monstre Jean Barbin fait plus que bousculer la norme, il la renverse, la met cul ar-dessus tête :

C'est le monde qui est à l'envers, ou c'est moi ?/ On n'est jamais à l'envers de soi-même./ Comment on s'en rendrait compte./ Donc c'est le monde qui est à l'envers./ C'est exactement ça, le grave problème du monde : il est à l'envers, et il ne s'en aperçoit pas./ Quand le monde est à l'envers, c'est vous qu'il veut remettre droit./ Alors que non, bien sûr que non, ce n'est pas vous qui êtes à l'envers./ L'envers de soi c'est soi, l'envers du monde ce n'est pas le monde./ Je dis et maintiens qu'à chacun de choisir son envers et son endroit, et que c'est un droit. (Bon, «Jean Barbin, A l'envers », Web, 09/08/2017)

Passant du monde à son verlan, le démon, Jean Barbin inflige à la vidéo une mue, qui opère par soustraction et retranchement plutôt que par addition.

\section{La pure écriture}

Dans cette écriture à voix haute, cette scription/diction (François Bon compare l'écrire à un " parler debout devant le film ») et même si, dans le cas des chroniques de Jean Barbin les textes sont pré-écrits, il s'agirait d'éditer, au minuit de soi, son monstre. Déformer la voix, les mots, pour retrouver la voix enfant : "Ceux qui chantent, ceux qui discourent, ceux qui crient sur toutes les scènes ont nié leur voix enfant./ Celui qui parle dans le noir cherche à tout effacer de ce qui n'est pas la voix enfant./ Vous l'entendez, ici, ma voix enfant? » (Bon, François, " Jean Barbin, La voix enfant », Web, 09/08/2017). nous confie Jean Barbin. Retrouver la voix des incipit ou des excipit de la vie, tendre l'écriture jusqu'à faire céder tout à la fois le genre narratif et poétique, déformer sa propre voix, la rendre étrange, pour se dévisager soi-même, et peutêtre, finalement, se reconnaître.

Ce que rend peut-être le plus visible Jean Barbin, dans sa monstration, c'est sans doute l'écriture même, à vif, à cru : « une vidéo qu'on parle dans la nuit s'appelle de l'écriture. De l'écriture qu'on « corps » dans la nuit s'appelle 
juste écriture ». Et puis se réveiller le matin après, ou devant, le monstre qu'on a été hier soir, " quel trouble de se consacrer à une telle écriture, ça vous met dans quel état, et bien difficile d'assumer les tâches connexes ». Il faut entendre sans doute sérieusement ce que dit l'auteur, qui ne se met pas dans un état émotionnel pour retrouver Jean Barbin, mais que l'écriture de Jean Barbin met dans cet état. Savoir en revenir, faire le voyage retour. Et quand même savoir qu'on a traversé, cette nuit, la rive. Un monstre à laisser en liberté la nuit peutêtre pour ne pas devoir le côtoyer en journée, dehors, dans la ville, lui accorder son temps, ses quelques minutes de gloire. Comme on sort les chiens malades la nuit, pour pas que les passants, pour pas la honte, et parce que la nuit les aime.

Trouver, par la vidéo nocturne, un autre écrire. Un écrire qui préserve le silence, et le non-né, le non-encore, le pas-déjà, dans l'avènement même, et que ce soit ça, l'événement. Jean Barbin se compare lui-même à une musique de nuit, une musique qui ne sort pas : « Dans cette nuit sous le ciel, marchant dans moi même j'entends une autre musique, lancinante, très construite " (Bon, François, "Jean Barbin, Musiques »). Quand l'écriture touche à cette précision des choses encore en gestation, à fleur plutôt qu'en fleurs. Quand elle fait du dehors avec du dedans.

Jean Barbin nous désarme parce que, pour se mettre à nu, il s'habille de noir. Pour être seul, il s'adresse à nous. Pour conjurer l'angoisse, il la parle. Un monstre confidentiel, dans un format confidentiel. Des vidéos faites dans la nuit, faites aussi peut-être pour la nuit, dans l'invisibilité du monde, pour l'entrenous, l' « antre »-monstres. Avec Jean Barbin, c'est YouTube en entier, comme espace d'écriture et de parole, qui se voit reconfiguré. Un internet qui crée ses pièces au fur et à mesure, les murs montent en même temps qu'on les parle. Et combien de monstres, dessous ? Les nuits recroquevillées, souterraines, de Jean Barbin secouent les flancs de YouTube et font pendant à l'écriture diurne, aux petits matins RER et aux longs travellings que l'auteur affectionne. Il y a bien le jour et la nuit, sur Internet. On retrouvera au matin, le lendemain, François Bon écrivant la ville, la filmant, parfois en live, en direct, sur Facebook, sorte de « Yamakazi » ou d' " artiste du déplacement », quand écrire la ville va avec marcher dans la ville.

A propos des chroniques de Jean Barbin, François Bon écrit : « Je ne les regarde plus jamais après une sorte d'instantané du dedans » (Bon, François, "Jean Barbin tout nu »). "Brûler les questions", écrivait Artaud dans L'Ombilic des Limbes. En trente-sept vidéos, et dans le noir, brûler une à une les questions. A bien y regarder, à mieux y regarder, c'est peut-être cela qu'on verrait : un feu. «Un jour, Jean Barbin ne sera plus du tout différent de moimême» écrit François Bon, sans qu'on sache bien qui des deux ira vers qui, et lequel sera l'envers ou l'endroit de l'autre.

\section{Bibliographie}

Bon, François. «La Chronique de Jean Barbin», Web, 09/08/2017.

Grossman, Simone. La Défiguration, Artaud, Beckett, Michaux. Paris :

Editions de Minuit, 2004.

Derrida, Jacques. « Forcener le subjectile. » Artaud, dessins, et portraits. Dir.

Paul Thévenin. Paris : Gallimard, 1986. 